\title{
LOGGING OF OPERATION DATA AT TLS
}

\author{
Tsai-Fu Lin, Yi-Chih Liu, Cheng-Kuo Chang, Hung-Chiao Chen, Ming-Jyi Horng, Jar-An Li, \\ Yao-Kwang Lin \\ 101 Hsin-Ann Road, Hsinchu Science Park, Hsinchu 30076, Taiwan, R. O. C.
}

\begin{abstract}
The Taiwan Light Source had been Top-up operation in October 2005 and the beam current had increased to $302 \mathrm{~mA}$ in early 2006. For normal operation, there are some important parameters of Top-up operation, such as beam stability, filling pattern and injection efficiency etc. These data have to be recorded and to be reference for the accelerator operation. Therefore, a LabVIEW-based data logging system had been developed. The system handles communication with other instruments via Ethernet and IEEE-488 interconnections. In this report, the design concept and the current status are described. The planned improvements are carried out in the future.
\end{abstract}

\section{INTRODUCTION}

The Top-up mode had been a general operation of Taiwan Light Source (TLS) in early 2006. The Top-up operation of TLS is that the injector inject electron beam to storage ring per minute and maintain the beam current at $302 \mathrm{~mA}$. Some essentials are important of the Top-up operation. They are such as injection efficiency, injector performance and status of injection sector. Therefore a data logging system had been developed. The system was developed by LabVIEW-based. It communicated with data base of accelerator control system via Ethernet. It also communicated with some digital oscilloscopes and spectrum analyzer via IEEE-488 interconnections. The system provides the archive data of operation in the main page. Moreover, the system recorded some parameters waveform of subsystems in per injection. The information is useful for adjusting subsystem setting. When subsystem of TLS malfunction that logging data is helpful for operator to debug [1-3].

\section{OPERATION DATA OF TOP-UP MODE}

The main page of data logging system presents some parameters of subsystem of TLS. Four figures of beam parameters are also shown in the main page. There are beam spectrum, filling pattern, injection efficiency and the beam current. The main page is shown in figure 1 .

\section{General Information}

The general information includes date, time, operation mode and operator on duty. The operation modes of TLS are decay mode and top-up mode. The lattice files were also shown in the main page. These files include the storage ring, booster synchrotron and transport line.

\section{Beam Parameters}

There are some beam parameters that show on the main page, such as beam current, beam lifetime and beam size. The difference of the root mean square (RMS) value between the real beam orbit and the reference orbit is also shown.

\section{Booster Parameters}

Some subsystem parameters of booster and linac (linear accelerator) are monitored. The cooling water temperature of linac is about $42^{\circ} \mathrm{C}$ and the water heater controlled by a PID controller. The input voltage of heater for cathode of electron gun is from $5 \mathrm{~V}$ to $5.8 \mathrm{~V}$ by aging of cathode. That voltage was tuned depend on the output of electron gun. Those are two important parameters to keep the output of electron gun and linac stable.

\section{Insertion Devices}

Three superconducting magnet insertion devices (SID) and four permanent magnet insertion devices (ID) are installed in the storage ring of TLS. The charge current of SID is constant. The betatron tune of storage beam will change when the magnet gap of insertion device increases or decreases. Operator should adjust the current setting of trim quadrupole magnet to keep the betatron tune in stable range.

\section{Feedback Systems}

There are three digital feedback systems applied in the storage ring for beam stability. They are global orbit feedback system, transverse feedback system and longitudinal feedback system. The main page shows the status of these feedback systems.

\section{Filling Pattern}

The harmonic number of TLS storage ring is 200. In general operation, the bucket address is from 20 to 148 . The filling pattern is monitored and the clean gap of pattern is counted. The clean gap of filling pattern keeps about 100ns.

\section{Beam Spectrum}

The beam spectrum is measured by HP 4396A spectrum analyzer. Operator could measure the betatron tune and synchrotron tune from the beam spectrum. The beam spectrum is helpful to judge whether the longitudinal feedback system is malfunction. 


\section{Injection Efficiency}

The highly injection efficiency means fewer beam loss in injection process. That also means a fewer dosage. The statistic of injection efficiency from transport line to storage was shown in main page. In the figure, the average efficiency and statistical dispersion (STD) of per user shift (8 hours) are also shown.

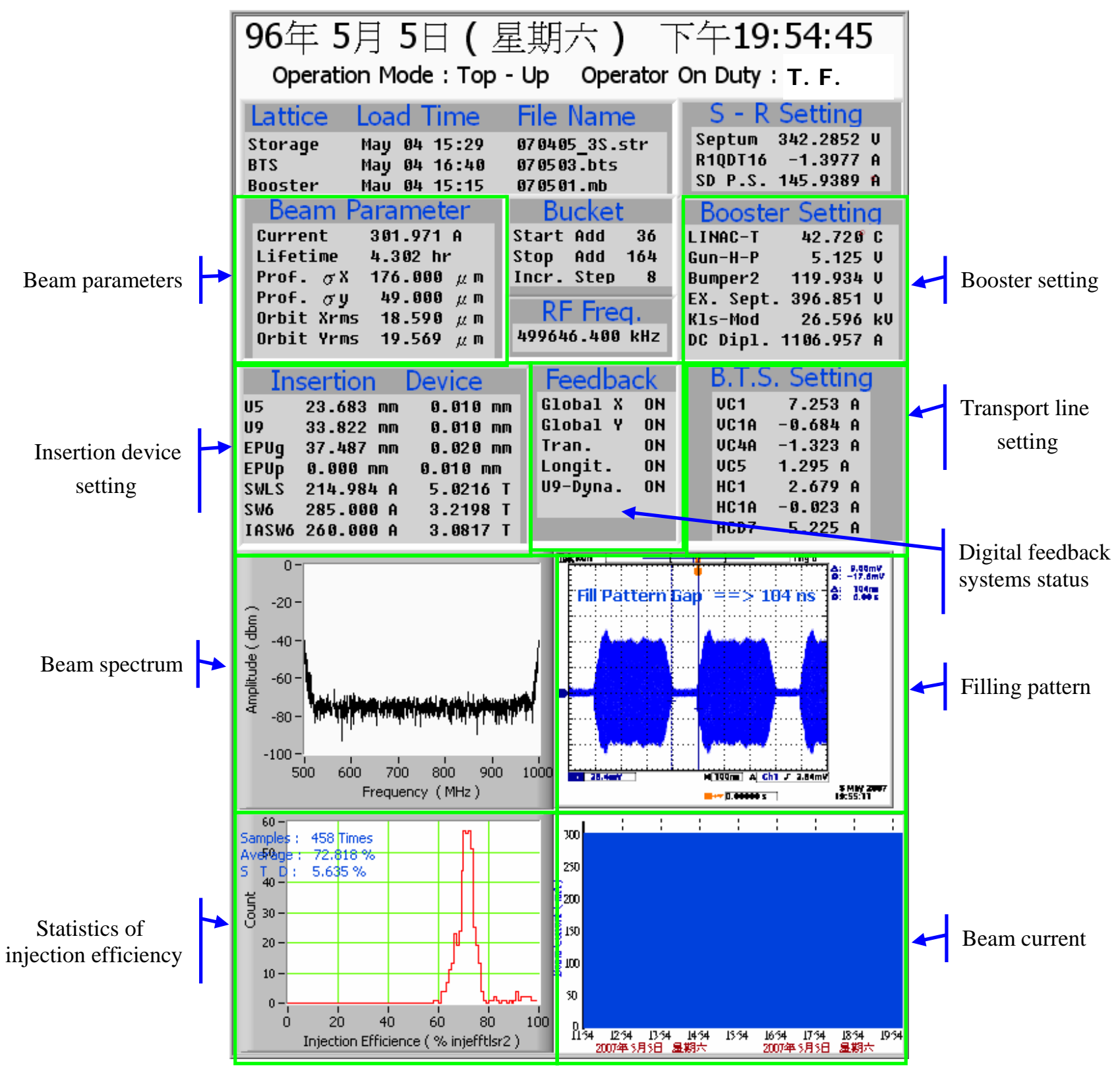

Figure 1: The main page of logging data

\section{INJECTION DATA RECORDS}

The other part of logging system was used to archive the data in per injection. That included the performance of pre-injector, booster current and waveform of kickers.

\section{Performance of pre-injector}

Three current transformers (CTs) are located at rear of electronic gun (CT1), front of linac (CT2) and rear of linac (CT3). The other current transformer (CT4) is behind of the 60 degrees bending magnet, which was on the transport line between the linac and booster. The waveform of the CT2 and CT3, i.e. the input and output of the linac, should be identical in the injection process. Moreover, the waveform of CT4 has to be similar to a rectangle wave. The highly reading of waveform of CT4 means a highly efficiency of pre-injector. Figure 2 shows the waveform of these current transformers. The width of waveform of CT4 is 40ns and the height is $150 \mathrm{mV}$. 


\section{Booster Current}

In Top-up operation, the current of booster synchrotron should be identical in injection process every time. The pre-injector and booster synchrotron adjustment could depend on the archive data of booster current before next injection. The data is also useful for operator to double check the injection efficiency. The booster current is shown in figure 3 . There are three shots in the injection process.

\section{Waveform of Kickers}

The waveform of injection kickers were monitored per injection. Sometimes, beam loss due to kicker misfire in top-up operation. The jitter of kicker power supply affects injection efficiency or generates partial beam loss at time. These records would identify the reason of the beam trip event helpfully. The waveform of kicker 1 to kicker 4 (channel 1 to channel 4) are shown in figure 4.

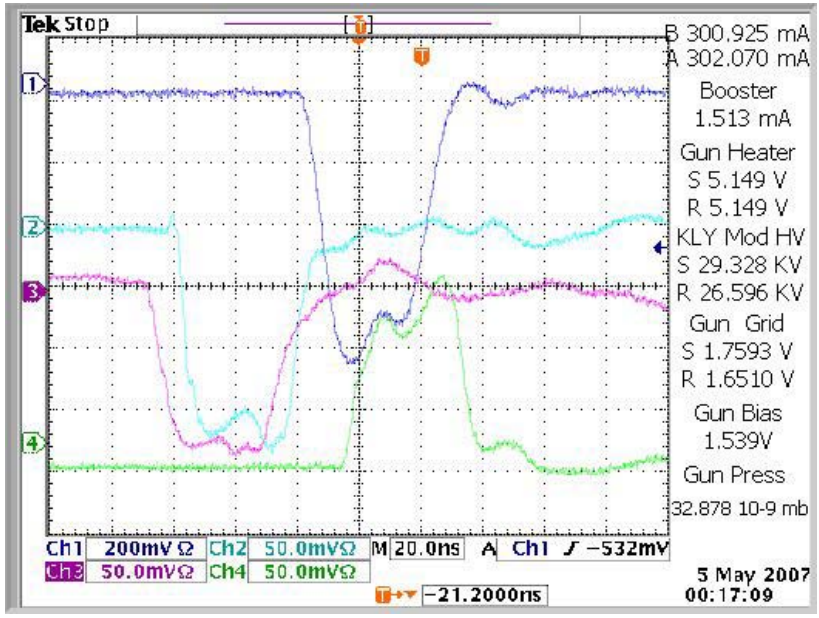

Figure 2: The reading of CT1 toCT4 (channel 1 to channel 4)

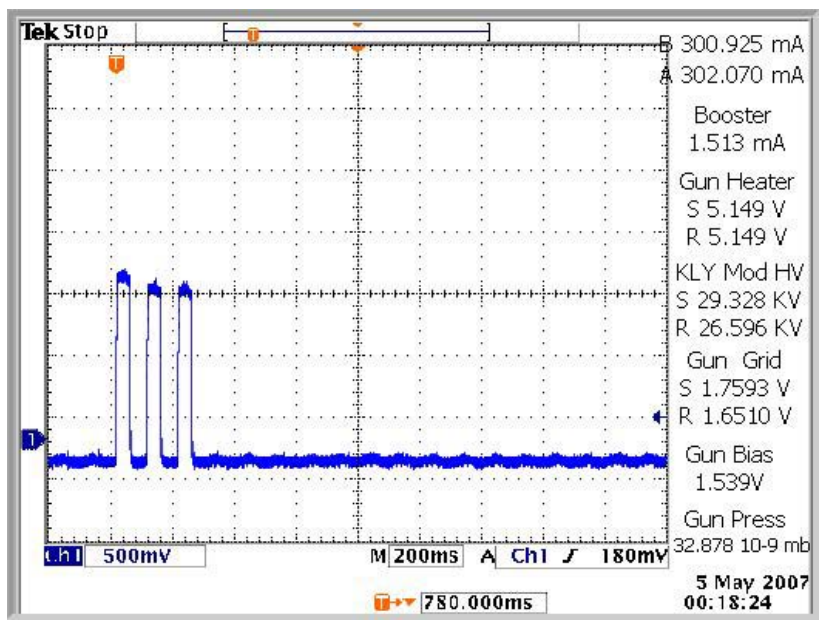

Figure 3: The booster current.

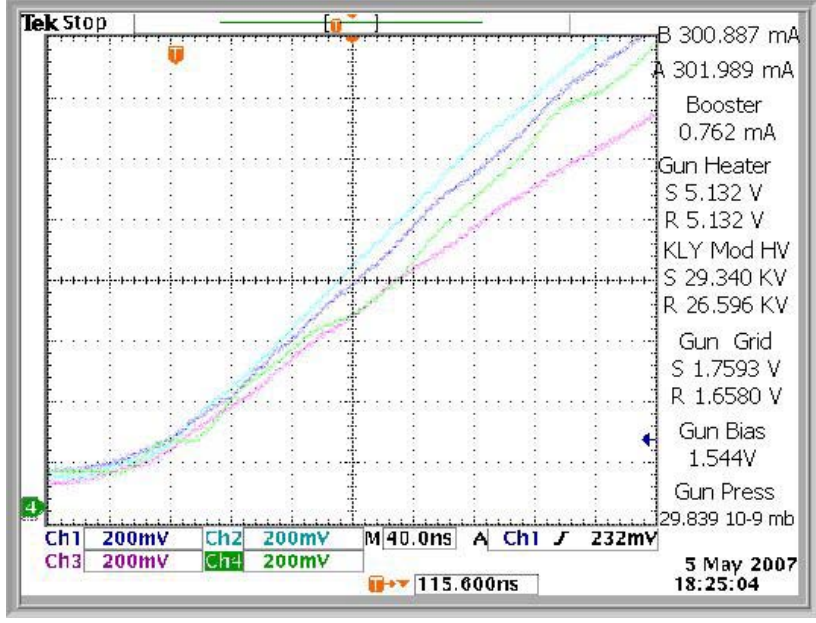

Figure 4: The waveform of injection kickers

\section{SUMMARY}

An operation data logging system was development for Taiwan Light Source operation. There are some advantages of this system:

1. The simply structure is easy to maintain and expand.

2. It is useful for operation to check the malfunction of accelerator subsystem.

3. The operation data recorded per injection would help operator adjust parameter of subsystem.

4. It is a base to develop an application program that depends on the archive data to adjust parameter automatically in future.

\section{REFERENCES}

[1] A. Chan, G. Crane, I. MacGregor and S. Meyer, "Data Warehouse on the Web for Accelerator Fabrication and Maintenance”, PAC’97, Vancouver, May 1997, p. 2413.

[2] T. Birke, R. Bakker, S. Bub, and R. Müller, "Logging of Supervisory Data at Bessy”, ICALEPCS'99, Trieste, Oct. 1999, p. 431.

[3] P. Klysubum and C. Netsai, "Development of Matlab-Based Data Logging System at Siam Photon Source”, EPAC’06, Edinburgh, Jun. 2006, p. 3098. 